\title{
Constructivismo y prácticas educativas: coherencia conceptual y de aplicación
}

\section{Constructivism and educational practices: conceptual and application coherence}

Recibido 25/09/2020

Aquilino Rocael Recinos

Universidad de San Carlos de Guatemala

rocaelrecinos@gmail.com

Aceptado 18/01/2021

\section{Referencia}

Recinos, A. R. (2021). Constructivismo y prácticas educativas: coherencia conceptual y de aplicación. Revista Docencia Universitaria, 2(1). 1-9. https://doi.org/10.46954/revistadusac.v2i1.19

\section{Resumen}

El objetivo de esta investigación es establecer la coherencia conceptual y de aplicación entre el constructivismo y las prácticas educativas. En el estudio participaron ocho profesores y doce estudiantes de posgrado en educación universitaria: los profesores son expertos en educación; los estudiantes cursaban el cuarto ciclo de la maestría y han egresado de diversidad de carreras. Se utilizó el enfoque mixto de tipo fenomenológico, con alcance descriptivo y diseño recurrente. Se aplicó dos instrumentos: a) cuestionario de preguntas abiertas: a los profesores, b) cuestionario de tipo escala de Likert: a los estudiantes. La información obtenida con el instrumento aplicado a los profesores se categorizó, en tanto que, los datos recolectados con el instrumento aplicado a los estudiantes se analizaron estadísticamente. Con base en los resultados, se evidenció que los profesores poseen una base conceptual clara acerca de los principales postulados del 
Palabras clave:

constructivismo, prácticas educativas, conocimientos previos, profesores, estudiantes
Keywords:

Constructivism, Educational practices, previous knowledge, professors, students constructivismo y de cómo debe aplicarse en las prácticas educativas. No obstante, se estableció que hay divergencia de percepción, pues los estudiantes notan que el constructivismo no es aplicado de acuerdo con la teoría. Los estudiantes manifestaron que en la mayoría de las prácticas educativas de los docentes se evade la activación de los conocimientos previos, lo cual incide en el proceso de aprendizaje, pues no se establece la conexión del conocimiento nuevo con el previo. La diferencia de parecer de profesores y estudiantes acerca de la activación de los conocimientos previos puede deberse a que las actividades propuestas tienen poca incidencia en el proceso de construcción de conocimientos.

\section{Abstract}

The objective of this research was to establish the conceptual and application coherence between Constructivism and Educational practices. In the study, eight professors and twelve graduate students in university Education participated: the professors are experts in education; the students were in the fourth cycle of the master's degree and have graduated from a diversity of careers. The Mixed phenomenological approach was used, with descriptive scope and recurrent design. Two instruments were applied: a) open-ended Questionnaire: to teachers, b) Likert scale-type Questionnaire: to students. The information obtained from the instrument applied to the teachers was categorized, while the data collected with the instrument applied to the students was statistically analyzed. Based on the results, it was evidenced that teachers have a clear conceptual basis about the main postulates of Constructivism and how it should be applied in Educational practices. However, it was established that there is a divergence of perception, since the students perceive that Constructivism is not applied according to the theory. The students state that in most of the Educational practices of the teachers, the activation of previous knowledge is avoided, which affects the learning process, since the connection of the new knowledge with the previous one is not established. The difference in opinion of teachers and students about the activation of prior knowledge may be due to the fact that the proposed activities have little impact on the knowledge construction process. 


\section{Introducción}

En la Escuela de Estudios de Postgrado, adscrita a la Facultad de Humanidades de la Universidad de San Carlos de Guatemala (FAHUSAC) se imparten maestrías y doctorados. De acuerdo con la información referida en el sitio web de la FAHUSAC, en la Escuela de Estudios de Postgrado se ofrecen las maestrías en Docencia Universitaria, en Investigación, en Currículum y en Letras, y los doctorados en Educación y en Filosofía (Facultad de Humanidades, s.f., “¿Qué carreras ofrece?”, párr. 1). La maestría en Docencia Universitaria fue el primer estudio de posgrado que se impartió en Escuela, a partir de 1983 (párr. 1).

Entre los estudios previos de estudiantes de las unidades académicas de la Universidad de San Carlos de Guatemala, hay algunas tesis de licenciatura que se relacionan con esta investigación, entre ellas: Estrategias de aprendizaje basado en el enfoque constructivista para docentes de aldeas infantiles, SOS, Santa Cruz del Quiché, Quiché, presentada por Angélica Judith Medina Sánchez en 2017 y la El modelo educativo constructivista en el proceso de enseñanza aprendizaje en la sede de Chiquimulilla de la Facultad de Humanidades de la Universidad de San Carlos de Guatemala, presentada por Siria Ileana González Reyes en 2015. Resulta llamativo que no se haya encontrado tesis de maestría sobre la aplicación del constructivismo.

Los enfoques educativos, pese a la amplia aceptación de la que gozan deben ser pasados por el tamiz de la investigación, con mayor razón en los estudios de posgrado en educación, ya que en estas carreras se forman expertos que deben manifestar una postura crítica ante las corrientes educativas en boga. En este sentido, reviste importancia establecer la coherencia conceptual del constructivismo y su aplicación en las prácticas educativas (actividades del profesor que ocurren antes, durante y después de la clase) de los profesores (García Cabrero et al., 2008), con el fin de establecer la correspondencia entre teoría y práctica de este enfoque educativo, teóricamente centrado en favorecer el proceso de construcción de aprendizajes de los estudiantes.

En esta investigación participaron doce estudiantes de la maestría en Docencia Universitaria y ocho profesores que les impartieron clases durante los cuatro ciclos académicos en las modalidades presencial y a distancia, esta última suscitada por la pandemia provocada por 
la COVID-19. De acuerdo con los resultados que se obtuvo mediante la aplicación de dos cuestionarios: uno de preguntas abiertas (profesores) y otro de tipo escala de Likert (estudiantes), se comprobó que los profesores tienen una comprensión conceptual clara acerca del constructivismo, lo cual se manifiesta en que las prácticas educativas que refieren, en su mayoría, tienen coherencia con este enfoque educativo. No obstante, profesores y estudiantes discrepan en cuanto a la percepción sobre la activación y el empleo de los conocimientos previos.

Este estudio carece de la intención de cuestionar al constructivismo. Tampoco se propone otro enfoque educativo. Lo que sí se pretende es motivar la reflexión pedagógica acerca del constructivismo y su aplicación. El análisis crítico de los enfoques educativos es una tarea necesaria en las carreras de posgrado en educación para obtener argumentos con base en resultados de investigaciones. Debido al diseño cualitativo, los resultados no se pueden generalizar, lo cual favorece que se efectúen más estudios acerca de la aplicación del constructivismo desde otros diseños, tipos y alcances de investigación.

\section{Materiales y métodos}

En la investigación se utilizó el enfoque mixto de tipo fenomenológico, con alcance descriptivo y diseño recurrente. Para la recolección de datos se aplicó dos instrumentos: un cuestionario de preguntas abiertas, a los profesores; un cuestionario de tipo escala de Likert, a los estudiantes. Para el análisis de los datos del cuestionario aplicado a los profesores se infirió categorías macro y meso (Vargas Beal, 2015). La información del cuestionario aplicado a los estudiantes se analizó estadísticamente. Se aplicaron criterios para la selección: estudios de licenciatura (estudiantes) y último grado académico (profesores), sin embargo, no se percibió incidencia en las respuestas.

\section{Resultados}

La descripción de la coherencia conceptual y de aplicación del constructivismo en las prácticas educativas se efectuó con base en la información obtenida por medio de la aplicación de los cuestionarios. Las respuestas del cuestionario de preguntas abiertas se clasificaron en dos categorías macro: a) concepción individual de constructivismo y b) proceso de construcción del conocimiento. 
En la primera categoría macro se identificó las categorías meso: a) definición de constructivismo, b) sujeto del constructivismo y c) objeto del constructivismo. La segunda categoría macro se dividió en las categorías meso: a) activación de conocimientos previos, b) mediación del aprendizaje, c) actividades para el aprendizaje, d) actividades de enseñanza y e) verificación del aprendizaje.

En la categoría macro concepción individual de constructivismo, las categorías meso que más indicadas por los profesores fueron: sujeto del constructivismo y objeto del constructivismo, en cuyas elecciones subyace la categoría meso: definición de constructivismo. Por otra parte, en la categoría macro proceso de construcción del conocimiento, los profesores señalaron con mayor frecuencia la categoría meso: actividades para el aprendizaje; la segunda categoría meso que más se identificó, aunque con menor frecuencia fue: mediación del aprendizaje.

En la tabla 1 se muestran las frecuencias de las categorías elegidas por los estudiantes (participantes) en el cuestionario propuesto para medir la percepción que ellos tienen acerca de cómo los docentes aplican el constructivismo en sus prácticas educativas, activan y usan los conocimientos previos. Las categorías fueron: nunca $(\mathrm{N})$, a veces (AV), sin evidencia (SE [opción neutral]), casi siempre (CS) y siempre (S).

\section{Tabla 1}

Frecuencias de categorías en las respuestas de los estudiantes

\begin{tabular}{|c|c|c|c|c|c|c|}
\hline \multirow[b]{2}{*}{ Preguntas } & \multicolumn{5}{|c|}{ Categorías } & \multirow[b]{2}{*}{ Participantes } \\
\hline & $\mathbf{N}$ & AV & SE & CS & $\mathbf{S}$ & \\
\hline $\begin{array}{l}\text { 1. ¿Usted percibe coherencia entre el constructivismo y las } \\
\text { prácticas educativas de sus docentes? }\end{array}$ & 1 & 6 & 0 & 4 & 1 & 12 \\
\hline 2. ¿Sus docentes activan conocimientos previos? & 1 & 5 & 0 & 6 & 0 & 12 \\
\hline 3. ¿Sus docentes provocan el conflicto cognitivo? & 1 & 8 & 0 & 3 & 0 & 12 \\
\hline $\begin{array}{l}\text { 4. ¿Sus docentes emplean estrategias para favorecer } \\
\text { la integración de los conocimientos previos con la } \\
\text { información nueva? }\end{array}$ & 0 & 7 & 0 & 5 & 0 & 12 \\
\hline
\end{tabular}

Fuente: propia. 
Nota. "El concepto de constructivismo del profesorado y su incidencia en las prácticas educativas", por Recinos, (s.f.), "Sistematización de datos".

La tabulación general de las categorías seleccionadas por la mayoría de los estudiantes muestra que, según ellos, los profesores activan con poca frecuencia los conocimientos previos. De estos resultados puede inferirse la poca incidencia de las actividades que los docentes proponen para activar y aprovechar los conocimientos previos. Aunque los resultados de este estudio no se pueden generalizar, el hecho de que una de las prácticas medulares del enfoque constructivista se haya ubicado en las categorías estadísticamente menos significativas puede ser considerado un llamado de atención sobre la aplicación del constructivismo.

Tabla 2

Resultados del análisis de las respuestas de los estudiantes

\begin{tabular}{|c|c|c|c|c|c|c|c|c|}
\hline \multirow{2}{*}{\multicolumn{2}{|c|}{ Preguntas }} & \multicolumn{5}{|c|}{ Categorías } & \multirow[b]{2}{*}{ Suma } & \multirow[b]{2}{*}{ Promedio } \\
\hline & & $\mathbf{N}$ & AV & SE & CS & $S$ & & \\
\hline 1. & $\begin{array}{l}\text { ¿Usted percibe coherencia entre } \\
\text { el constructivismo y las prácticas } \\
\text { educativas de sus docentes? }\end{array}$ & 1 & 12 & 0 & 16 & 5 & 34 & 2.83 \\
\hline 2. & $\begin{array}{l}\text { ¿Sus docentes activan conocimientos } \\
\text { previos? }\end{array}$ & 1 & 10 & 0 & 24 & 0 & 35 & 2.92 \\
\hline 3. & $\begin{array}{l}\text { ¿Sus docentes provocan el conflicto } \\
\text { cognitivo? }\end{array}$ & 1 & 16 & 0 & 12 & 0 & 29 & 2.42 \\
\hline 4. & $\begin{array}{l}\text { ¿Sus docentes emplean estrategias } \\
\text { para favorecer la integración de } \\
\text { los conocimientos previos con la } \\
\text { información nueva? }\end{array}$ & 0 & 14 & 0 & 20 & 0 & 34 & 2.83 \\
\hline
\end{tabular}

Nota. "El concepto de constructivismo del profesorado y su incidencia en las prácticas educativas", por Recinos, (s.f.), "Sistematización de datos". 
En la tabla 2, se detalla los resultados que se obtuvo tras el análisis estadístico de las frecuencias (ver tabla 1). A cada categoría se le asignó un valor en la escala 1-5: $N=1, A V=2, S E=3, C S=4, S=5$. La frecuencia se multiplicó por el valor asignado a la categoría. Después se sumó horizontalmente las categorías de cada pregunta, el resultado de la suma se dividió dentro de 12 (participantes) para obtener el promedio. Las respuestas se ubican en la categoría SE, pues están en el rango 2.01-3.00.

Pese a que los profesores afirmaron en el cuestionario que sí activan los conocimientos previos, los estudiantes perciben de manera distinta las prácticas educativas de los docentes. En la tabla 2, que contiene el análisis estadístico que toma en cuenta las respuestas de todos los participantes (estudiantes), se confirma la tendencia mostrada en la tabla 1: las actividades que los docentes plantean para activar los conocimientos previos son poco relevantes para el proceso de construcción de conocimientos: todas las preguntas se ubican en la categoría SE: es decir, la opción neutral. Estos resultados cuestionan la aplicación del constructivismo por parte de los docentes, en una de sus prácticas fundamentales: la activación y uso de los conocimientos previos.

\section{Discusión}

La teoría sobre el constructivismo refiere que la activación de conocimientos previos es una práctica educativa necesaria para que los estudiantes integren el conocimiento previo con el nuevo (Miras, 1999). El cruzamiento de la información recolectada mediante los cuestionarios muestra discrepancia en la percepción de los profesores y los estudiantes acerca de la coherencia entre el enfoque constructivista y la aplicación en las prácticas educativas de los docentes, referidas a la activación y uso de los conocimientos previos.

Por su parte, los profesores expresan claridad conceptual sobre el constructivismo, pues manifiestan que en las clases promueven, median el aprendizaje y activan y usan los conocimientos previos. Empero, los resultados del cuestionario aplicado a los estudiantes reflejan que ellos no perciben coherencia conceptual y de aplicación del constructivismo en las prácticas educativas de los profesores relacionadas con la activación y el empleo de los conocimientos previos, mediante estrategias diversas. La mayoría señala que esta actividad inicial es efectuada por algunos profesores. 
El hecho de que el promedio de todas las preguntas planteadas a los estudiantes se haya ubicado en la categoría SE (sin evidencia), manifiesta que, en la opinión general de los alumnos, la activación y el uso de los conocimientos previos por parte de los profesores tiene una influencia poco relevante en el diseño de las clases y en el proceso de aprendizaje de los alumnos. Es decir, los conocimientos previos no constituyen una práctica educativa fundamental en las experiencias de aprendizaje que proponen los profesores: una contradicción conceptual y de aplicación del constructivismo.

\section{Referencias}

Facultad de Humanidades. (s.f.). Escuela de Estudios de Postgrado. http://www.humanidades.usac.edu.gt/usac/postgrado/

García Cabrero, B., Loredo Enríquez, J., y Carranza Peña, G. (2008). Análisis de la práctica educativa de los docentes: pensamiento, interacción y reflexión. Revista Electrónica de Investigación Educativa, 10(especial), 1-15. http://www.scielo.org.mx/scielo. php?script=sci_arttext\&pid=S1607-40412008000300006\&lng =es\&tlng=es

Miras, M. (1999). Un punto de partida para el aprendizaje de nuevos contenidos: los conocimientos previos. En C. Coll, E. Martín, T. Mauri, M. Miras, J. Onrubia, I. Solé y A. Zabala, El constructivismo en el aula (9. ${ }^{a}$ ed., pp. 47-64). Graó. http://www.terras.edu.ar/ biblioteca/3/3Disponibilidad-del-aprendizaje.pdf

Recinos, A. R. (s.f.). El concepto de constructivismo del profesorado y su incidencia en las prácticas educativas [tesis de maestría, sin presentar; Universidad de San Carlos de Guatemala].

Vargas Beal, X. (2015). Paso 43: construcción de categorías [video]. YouTube. https://youtu.be/JDoCyU8dxZ8 


\section{Sobre el autor}

Es licenciado en Educación y Aprendizaje por la Universidad Rafael Landívar y tesista de la maestría en Docencia Universitaria y de la licenciatura en Letras, cursadas en la FAHUSAC. Ha impartido docencia en los niveles básico, diversificado y universitario. Actualmente trabaja como profesor del curso de estilo APA en la Facultad Latinoamericana de Ciencias Sociales (Flacso), sede Guatemala, y como corrector de estilo de tesis en la Facultad de Ingeniería de la Usac.

Copyright (c) Aquilino Rocael Recinos

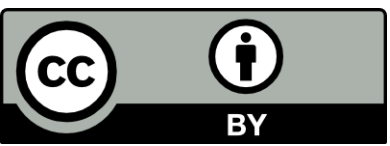

Este texto está protegido por una licencia Creative Commons 4.0.

Usted es libre para compartir, copiar y redistribuir el material en cualquier medio o formato y adaptar el documento, remezclar, transformar y crear a partir del material para cualquier propósito, incluso comercialmente, siempre que cumpla la condición de atribución: usted debe reconocer el crédito de una obra de manera adecuada, proporcionar un enlace a la licencia, e indicar si se han realizado cambios. Puede hacerlo en cualquier forma razonable, pero no de forma tal que sugiera que tiene el apoyo del licenciante o lo recibe por el uso que hace. 